\title{
Intestinal schistosomiasis of ljinga Island, north-western Tanzania: prevalence, intensity of infection, hepatosplenic morbidities and their associated factors
}

\author{
Andreas Mueller ${ }^{1,2^{*}+}$, Antje Fuss ${ }^{2+}$, Uwe Ziegler ${ }^{1}$, Godfrey M. Kaatano ${ }^{3}$ and Humphrey D. Mazigo ${ }^{4}$
}

\begin{abstract}
Background: Intestinal schistosomiasis is highly endemic in Tanzania and mass drug administration (MDA) using praziquantel is the mainstay of the control program. However, the MDA program covers only school aged children and does not include neither adult individuals nor other public health measures. The ljinga schistosomiasis project examines the impact of an intensified treatment protocol with praziquantel MDA in combination with additional public health interventions. It aims to investigate the feasibility of eliminating intestinal schistosomiasis in a highly endemic African setting using an integrated community-based approach. In preparation of this project, we report about baseline data on S.mansoni prevalence, intensity of infection, related hepatosplenic morbidities and their associated factors.
\end{abstract}

Methods: A cross sectional study was conducted among 930 individuals aged 1-95 years living at ljinga Island, north-western Tanzania in September 2016. Single stool and urine samples were collected from each study participant and processed using Kato Katz (KK) technique and point-of-care Circulating Cathodic (POC-CCA) antigen test for detection of S.mansoni eggs and antigen respectively. Ultrasonographical examination for S.mansoni hepatosplenic morbidities was done to all participants. For statistical analyses Fisher's exact test, chi-square test, student-t-test, ANOVA and linear regression were used where applicable.

Results: Overall based on KK technique and POC-CCA test, 68.9\% (95\%Cl: 65.8-71.8) and 94.5\% (95\%Cl: 92.8-95.8) were infected with S.mansoni. The overall geometrical mean eggs per gram (GMepg) of faeces was $85.7 \mathrm{epg}(95 \% \mathrm{Cl}$ : 77.5-94.8). A total of 27.1, 31.2 and 51.9\% of the study participants had periportal fibrosis (PPF-grade C-F), splenomegaly and hepatomegaly. Risk factors for PPF were being male $(\mathrm{aRR}=1.08,95 \% \mathrm{Cl}: 1.02-1.16, P<0.01)$, belong to the age group $16-25$ years ( $a R R=1.23$, 95\%Cl: $105-1.44, P<0.01), 26-35$ years $(a R R=1.42,95 \% \mathrm{Cl}: 1.21-$ $1.67, P<0.001), 36-45$ years (aRR $=1.56,95 \% \mathrm{Cl}: 1.31-1.84, P<0.001)$ and $\geq 46$ years (aRR $=1.64,95 \% \mathrm{Cl}: 1.41-1.92, P<$ 0.001). The length of the left liver lobe was associated with being female $(P<0.03)$, belong to the age group $1-5$ years $(P<0.013), 6-15$ years $(P<0.04)$ and S.mansoni intensity of infection $(P<0.034)$. Male sex $(a R R=1.15,95 \% \mathrm{Cl}$ : $1.06-1.24, P<0.001$ ) and belonging to the age groups $16-25$ years (aRR $=1.27,95 \% \mathrm{Cl}: 1.05-1.54, P<0.02$ ) or $26-35$ years $(a R R=1.32,95 \% \mathrm{Cl}: 108-1.61, P<0.01)$ were associated with splenomegaly.

\footnotetext{
* Correspondence: andreas.mueller@kwm-klinikum.de

${ }^{\dagger}$ Andreas Mueller and Antje Fuss contributed equally to this work.

'Department of Tropical Medicine, Klinikum Wuerzburg Mitte gGmbH,

Medical Mission Hospital, Salvatorstrasse 7, 97074 Wuerzburg, Germany

${ }^{2}$ Medical Mission Institute, Salvatorstrasse 7, 97074 Wuerzburg, Germany

Full list of author information is available at the end of the article
}

(c) The Author(s). 2019 Open Access This article is distributed under the terms of the Creative Commons Attribution 4.0 International License (http://creativecommons.org/licenses/by/4.0/), which permits unrestricted use, distribution, and reproduction in any medium, provided you give appropriate credit to the original author(s) and the source, provide a link to the Creative Commons license, and indicate if changes were made. The Creative Commons Public Domain Dedication waiver (http://creativecommons.org/publicdomain/zero/1.0/) applies to the data made available in this article, unless otherwise stated. 
(Continued from previous page)

Conclusion: Schistosoma mansoni infection and its related morbidities (hepatomegaly, splenomegaly, periportal fibrosis) are common in the study area. Age, sex and intensity of infection were associated with periportal fibrosis. The prevalence of S.mansoni was above 50\% in each age group and based on the observed prevalence, we recommend MDA to the entire community.

Keywords: Schistosoma mansoni, Prevalence, Hepatosplenic, ljinga, Tanzania

\section{Background}

Schistosomiasis is a neglected tropical disease which is highly prevalent in sub-Saharan Africa. An estimated 93\% of the approximated 290 million people affected by the disease worldwide are living in the region [1-3]. Approximately, 120 million people have schistosomiasis related symptoms and the disease accounts for over 2.8 million years lived with disabilities [2]. In the region of sub-Saharan Africa, schistosomiasis is due to Schistosoma mansoni causing intestinal schistosomiasis and $S$. haematobium causing urogenital schistosomiasis [4]. Intestinal schistosomiasis caused by Schistosoma mansoni remains an important public health concern in SubSaharan Africa and is associated with significant morbidities [3-5]. Chronic S. mansoni infection is associated with hepatosplenic disease characterized by hepatomegaly, splenomegaly and progressive periportal fibrosis which can lead to portal hypertension, esophageal varices, liver surfaces irregularities, portal-systemic venous shunts and haemetemesis [3, 6, 7]. Many of these $S$. mansoni related morbidities can be detected and measured by ultrasonography and classified according to the World Health Organization grade scales using the Niamey protocol $[8,9]$.

Available evidence indicates that an estimated 8.5 million cases of chronic hepatosplenic schistosomiasis are attributed to $S$. mansoni infection in sub-Saharan Africa [3, 4]. The underlying pathophysiology is the immunological response to S. mansoni eggs which are carried to the liver by the blood stream. The eggs are trapped in the liver tissue around branches of the portal vein and provoke an inflammatory response mediated by $\mathrm{CD}^{+}{ }^{+} \mathrm{T}$-lymphocytes. Finally, this results in small fibrotic scars and over time it leads to periportal fibrosis. This process is considered to be the main pathogenetic process of portal hypertension and other hepatosplenic complications of schistosomiasis due to S. mansoni [7, 10]. In contrast the habitat of adult $S$. haematobium are the venous plexus of the urogenital organs which do not drain into the portal vein. S. haematobium is not associated with periportal fibrosis therefore. It is worth noting that epidemiological and demographic factors such as duration of residence in endemic areas [11, 12], involving in fishing activities [12], parasitological factors (low versus heavy infection intensities) [13], age (children versus adults) [5] and genetic factors [14] can contribute to development of hepatosplenic disease in endemic populations.

The World Health Organization data indicate that after Nigeria, Tanzania has the highest number of schistosomiasis cases in sub Saharan Africa [15]. In 2012 it was estimated that about $52 \%$ of the Tanzanian population (23 million people) are infected with schistosomiasis $[15,16]$. The projected population of Tanzania currently stands at 55 million people [17]. In Tanzania, schistosomiasis is caused by $S$. mansoni and S. haematobium [5]. Schistosoma mansoni is focally distributed and commonly found along the large water bodies such as the Lake Victoria [5]. Indeed, multiple studies indicate, that the species $S$. mansoni is the by far predominant cause of schistosomiasis along the shoreline of Lake Victoria and on its islands $[5,12,13,16,18,19,20,21,22]$. Communities living there are disproportionally highly affected by the $S$. mansoni-specific pathology and a number of studies have reported a high prevalence of hepatosplenic morbidities in this region $[5,12,13,23$, 18]. A study among 360 school children on Ukerewe Island in Lake Vicctoria found splenomegaly in 90,7\%, right liver lobe hepatomegaly in $89 \%$ and overt signs of periportal fibrosis in $5,4 \%$ of the participants [5]. The disease is highly common among school children and adults [19, 24], of recent, data have emerged showing that pre-school aged children are also infected and carry heavy infection intensities [20].

At present, the main control approach against intestinal schistosomiasis in Tanzania focuses on mass drug administration (MDA) with praziquantel [5]. The MDA approach mainly targets school aged children (SAC) or children attending primary schools and drugs are offered at the school environment [25]. This approach has been advocated as very cost-effective, especially when drugs are distributed irrespective of the infection status. Repeated rounds of MDA have been reported to result in reduction of prevalence of infection, intensity of infection and reversibility of enlarged organs [25, 26, 27]. It should be noted that despite the implementation of several rounds of annual MDA in Tanzania, the prevalence of the disease remains very high in many areas surrounding Lake Victoria $[5,19,20,21]$. It is unlikely that a control strategy based on MDA alone will result into 
the interruption of transmission and elimination of the disease in high prevalence areas. Understanding this, World Health Assembly (WHA) in its 2012 resolution 65.21 [14] called for integrated control approaches which are mainly characterised by inclusion of public health education, access to clean water, improved sanitation and snail control into the MDA program [24]. Following this concept, we launched a pilot study on Ijinga island in Lake Victoria where we combine an intensified MDA program with repeated health education and WASH interventions. In preparation of this 5-year project, we collected baseline data which will be useful for monitoring the impact of the implemented intervention measures at the study area. Thus, the present study reports data on $S$. mansoni prevalence, intensity of infection, related hepatosplenic morbidities and their associated factors.

\section{Methods}

\section{Study area}

The present study was conducted at Magu district, specifically on Ijinga Island located on the southern shore of the Lake Victoria in north-western Tanzania. Ijinga Island and Magu district are located at $2^{\circ}$ South of the equator and $33^{\circ}-34^{\circ}$ East. The area is characterized by a tropical type of climate with bimodal rain seasons, short rain season running from October to December and long rain seasons starting from March to May. The average annual temperature is $26.5^{\circ} \mathrm{C}$, average annual rainfall is $1200 \mathrm{~mm}$ [28]. The island has a total of 420 households and is inhabited by a total of 2520 permanent residents. The majority of the inhabitants are involved in subsistence farming, fishing and livestock keeping. In the area the main source of water for domestic and recreational use for the inhabitants is Lake Victoria. Because of the high water contact levels, inhabitants have a high risk of becoming infected with $S$. mansoni and the high exposure maintains high intensities of the infection into adulthood. Annual mass drug administration (MDA) using praziquantel is mainly conducted among school children within the school environment, other community members are not involved, especially adult and non-school going children (pre-school children and other age groups out of the school).

\section{Study design, sample size, inclusion and exclusion criteria} The study was designed as analytical cross-sectional survey conducted between September and October 2016 on Ijinga Island. The study included all individuals aged from 1 year, who were permanent inhabitants of the study area as per our house to house census and gave consent/assent for participation in the study. The sample size was determined using a formula for binary outcome (S. mansoni infection) in a cross-sectional study design, developed by Leslie Kish [29]: $\mathrm{n}=\mathrm{z}^{2} \mathrm{p}(1-\mathrm{p}) / \mathrm{d}^{2}$ ( $\mathrm{n}=\mathrm{sam}-$ ple size, $\mathrm{z}=$ level of confidence, $\mathrm{p}=$ expected prevalence in proportion of one, $d=$ precision in proportion of one). For the conventional level of confidence of $95 \%$ the $\mathrm{Z}$ value was 1.96. Considering the prevalence of $S$. mansoni of $47.8 \%$ [23], in four villages along the shore of Lake Victoria, at 95\% confidence interval and margin errors of $3.2 \%$, the minimum sample size required was 936 . Considering $10 \%$ of refusal and non-responding (addition of 94 participants), a total sample size of 1030 was required for this study. A few days prior to the study a team of community health care workers visited all households for individual counselling and invited all permanent residents to take part. Participation was voluntarily and entirely free of charge. All inhabitants of the island who wished to be examined and did not receive Praziquantel during the last 3 months were eligible for participation. The investigations were carried out during an entire week including the weekend allowing also the working population to take part in the study and to minimize a sampling bias. A total of 930 participants participated voluntarily in this present study. Despite the fact that the sample size was below the minimum sample size, the power of the study remained above $90 \%$.

\section{Data collection}

(i) Interview using questionnaire

A pre-tested questionnaire was used to collect demographic information from study participants (age, sex, and residence). The history of participations in the mass drug administration program was recorded from school aged children, adolescents and adults. For adult participants additional questions on economic activities were included.

(ii) Parasitological examination of Schistosoma mansoni eggs using Kato Katz technique

From each study participant, a single stool sample was collected using a labelled stool container and from each collected sample two Kato Katz thick smears were prepared using the Kato Katz technique with a template of $41.7 \mathrm{mg}$ per thick smear [30]. Two experienced laboratory technicians examined all the Kato Katz thick smears at the National Institute for Medical Research, Mwanza, Tanzania. For quality assurance, $15 \%$ of all positive and negative Kato Katz slides were re-examined by a third independent laboratory technician who was blinded of the results of the first two laboratory technicians.

(iii) Examination of Schistosoma mansoni Circulating Cathodic Antigen (CCA) test in urine samples 
A point-of-care Circulating Cathodic Antigen (CCA) test (Rapid Medical Diagnostic- http://www.rapid-diagnostics.com/, batch number 50182) was used to screen for CCA antigen in urine samples. A single urine sample was collected from each of the study participants and examined for CCA antigen based on the manufacturer's instructions (Rapid Medical Diagnostic- http://www.rapid -diagnostics.com/). Trace results of the test were considered as positive. Laboratory technicians trained on POCCCA test and with experience from previous studies performed the test. The entire laboratory team involved in CCA examination was blinded of the Kato Katz results.

\section{(iv) Ultrasound examinations of Schistosoma mansoni} related morbidities

All study participants were clinically examined for consistency of the liver and the spleen using the approach described elsewhere [31]. Three ultrasonographers (medical doctors with long experience of ultrasound examination) participated in the ultrasonographical examination of the study participants. For quality assurance, $10 \%$ of the study participants were reexamined by all three ultrasonographers at different points and the results of the participants were agreed between them. In case of any inconsistence/disagreement, participants were requested to be re-examined. Classification of the ultrasound detected S. mansoni related morbidities was done using the modified Niamey Protocol [8]. The spleen size, peripheral portal branches (PPBs), periportal fibrosis, liver texture patterns, thickness of the peripheral portal branches were classified based on the Niamey protocol [8]. The degree of periportal fibrosis was qualitatively classified based on the liver image patterns categorized as A, B, C, D, E and F as per Niamey protocol $[8,17]$. The liver image/texture patterns $\mathrm{A}$ and $\mathrm{B}$ were classified as normal.

The size of the left liver lobe was measured along the right para-sternal line (PLL). For the spleen size, the longest diameter was taken along the craniocaudal axis of the spleen from the left flank side. The portal vein diameter (PVD) was measured at a midway between the confluence of the splenic vein and the superior mesenteric vein and its bifurcation inside the liver. The length of the left liver lobe, spleen and the diameter of the portal vein were adjusted using the height data from the reference population [8].

\section{Data analysis}

All collected data were double entered into an excel sheet, cleaned and imported to Stata Version 15 (StataCopr, 2017, Statistical software, College Station, TX: StataCorp LP. Texas, USA). All categorical variables were summarized using percentages and comparison of categorical/ proportions variables either using Fisher's exact or chisquare tests $\left(x^{2}\right)$. All continuous variables were analyzed using univariate test and reported as means or median with their standard deviation or interquartile ranges depending on the nature of distributions of the continuous variables. The Geometrical mean eggs per gram of feaces (GMepg) for S. mansoni eggs were calculated for each study participant from the non-logarithmically transformed means. Comparison of mean eggs count for $S$. mansoni between different demographic characteristics of the study participants was done using student-t-test (two groups) or ANOVA (more than two groups). Intensity of infection was categorized as 1-99 epg (low), 100-399 epg (moderate), $\geq 400 \mathrm{epg}$ (heavy) intensities [32]. The ultrasound height adjusted data for the liver, spleen and portal vein diameter were compared between the infected and uninfected participants using the t-test. The Niamey protocol was used to define the cut-off point between normal and enlarged spleen, left liver lobe and dilatation of the portal vein dilatation [8].

Generalized linear model (glm) and the linear regression model were used to assess the contributions of intensities of $S$. mansoni, age, sex and infection status on the extent of the liver and spleen enlargements. The glm was used to determine risk factors associated with periportal fibrosis and splenomegaly while the linear regression model was used to assess predictors of increased height of the left liver lobe. Linear regression model was constructed for heightadjusted measurements of the left liver lobe and the above mentioned explanatory variables were included. At bivariate analysis, explanatory variables with $P$ value $<0.2$ were considered for multivariable analyses. For the glm, Risk Ratios with their 95\%CI were generated and used to measure the level of risk and the Beta coefficients with their corresponding $95 \% \mathrm{CI}$ were also generated from the linear regression. The $P$ values of $<0.05$ were considered significant.

\section{Treatment}

All participants who were diagnosed with S. mansoni infection were treated with a single dose of praziquantel (40 mg/kgBwt). Treatment was done under direct observation (DOT) of qualified nurses. All study participants received food before taking the drug to minimize possible side effects of praziquantel. After taking drugs, participants were under direct observation for two hours before leaving the treatment area and asked to report any side effects to the study team. In case of abdominal pain, symptomatic treatment with paracetamol and nbutylscopolamin was used. Children aged $<6$ years received crushed tablets mixed with juice according to their body weight. 


\section{Results}

\section{Characteristics of the study participants}

A total of 930 participants were enrolled in the present study, of these $513(55.2 \%)$ and 417 (44.8\%) were female and male respectively. The mean age of the study participants was $23.3 \pm 18.5$ years. Of the study participants $87.2 \%$ reported to have never participated in a mass drug administration campaign against schistosomiasis. Only $12.8 \%$ reported to have participated at least once in the program. Table 1 shows the age and sex distribution of the study participants. All participants were from Ijinga Island, the majority of the adult participants reported to be involved in subsistence farming, livestock keeping and fishing.

\section{Prevalence and intensity of infection of Schistosoma mansoni using Kato Katz technique}

The overall prevalence of S. mansoni was $68.9 \%$ (95\%CI: 65.8-71.8). The prevalence of $S$. mansoni among male individuals was $74.3 \%$ (95\%CI: 69.9-78.3) and $64.5 \%$ (95\%CI: 60.3-68.6) in female participants. This gender difference was significant $\left(\chi^{2}=10.3523, P<0.001\right)$. In the age group of 6-15 years the prevalence of $S$. mansoni was $86.1 \%$ (95\% CI: 82.4-89.1) and among the 16-25 years old the prevalence was $57.1 \%$ (95\%CI: 47.9-65.8). Compared to the other age groups these 2 age groups of $\mathrm{SAC}$, adolescents and young adults had a significantly higher prevalence of $S$. mansoni $\left(X^{2}=105.61, P<0.001\right)$. Table 2 shows the prevalence of $S$. mansoni categorized by sex and age.

The overall geometrical mean eggs per gram of faeces (GMepg) was 85.7epg (95\%CI: 77.5-94.8), with no sex differences (89.9GMepg for male versus 81.4GMepg for female, $\mathrm{t}=-0.2887, P=0.77)$. There was a significant difference in GMepg between the age groups 1-5 years (91,9GMepg, 95\% CI:58.8-143.5) and 6-15 years (108.6GMepg, 95\% CI:95.2-124.0) (F-test $=10.71, P<$ $0.0001)$. The age group $6-15$ years had the highest intensity of infection among all age groups. Table 3 shows the Geometrical mean eggs per gram of faeces of S. mansoni stratified by sex and age. Based on the WHO classification of intensity of infection, majority of the study participants had light $(55.2 \%)$ to moderate $(20.4 \%)$ intensity of infection. Only $12.9 \%$ of them had a heavy intensity of infection.
Prevalence of Schistosoma mansoni based on circulating cathodic antigen tests

The laboratory results for the point-of-care Circulating Cathodic Antigen (POC-CCA) tests were available for 929 study participants. The overall prevalence of S. mansoni based on the POC-CCA test was 94.5\% (95\%CI: 92.8-95.8). The male individuals recorded the highest prevalence of $S$. mansoni compared to female individuals (96.2\% versus $\left.93.2 \%, X^{2}=3.9839, P=0.046\right)$. In relation to age groups, the age groups $6-15$ years recorded the highest prevalence of infection compared to other age groups (98.1\%, $\left.X^{2}=34.2723, P<0.001\right)$. Table 4 below shows the prevalence of $S$. mansoni infection based on POC-CCA test stratified by age and sex.

\section{Prevalence of periportal fibrosis and associated factors}

The overall prevalence of definite periportal fibrosis (PPF) (grade C-F) was 27.1\% (95\%CI: 24.3-30.1). 58,2\% of the participants showed a normal liver pattern (grade A), $14,7 \%$ a grade B pattern, which was considered as not distinct for PPF according to Kaatano et al. [33] and King et al. [34]. In relation to sex of the study participants, the prevalence of PPF was higher in male individuals than in female individuals $(P<0.026)$. In general, the oldest age groups, $36-45$ years and $\geq 46$ years had a higher prevalence of PPF compared to other age groups $(P<0.001)$. In relation to infection status, the findings indicate that individuals who had no $S$. mansoni detectable eggs in their stool samples recorded the highest prevalence of PPF $(P<0.005)$. The findings indicated that there was no significant difference in PPF prevalence in relation to intensity of infection categories $(P=$ 0.42). Table 5 shows the prevalence of periportal fibrosis in relation to demographic characteristics of the study participants, infection status and intensity of infection.

At bivariate analysis, being a male, belonging to age groups $16-25$ years, $26-35$ years, $36-45$ years and $\geq 46$ years were associated with periportal fibrosis (Table 6). On multivariable analysis the following variables were associated with periportal fibrosis (Table 6):

a) being male $(\mathrm{aRR}=1.08,95 \% \mathrm{CI}: 1.02-1.16, P<0.01)$,

b) belonging to the age group $16-25$ years $(\mathrm{aRR}=1.23$, 95\%CI: $1.05-1.44, P<0.01)$,

c) belonging to the age group $26-35$ years $(\mathrm{aRR}=1.42$, 95\%CI: $1.21-1.67, P<0.001)$,

Table 1 Age and sex distribution of the study participants on ljinga Island, north-western Tanzania

\begin{tabular}{lllllll}
\hline Sex & \multicolumn{5}{l}{ Age groups (in years) } & \\
\cline { 2 - 6 } & $1-5$ & $6-15$ & $16-25$ & $26-35$ & $36-45$ & $\geq 46$ \\
\hline Female & $45(59.2 \%)$ & $213(50.9 \%)$ & $65(54.6 \%)$ & $55(53.4 \%)$ & $65(71.4 \%)$ & $73(57 \%)$ \\
Male & $29(40.8 \%)$ & $205(49 \%)$ & $54(45.4 \%)$ & $48(46.6 \%)$ & $26(28.6 \%)$ & $55(42.9 \%)$ \\
Total & 71 & 418 & 119 & 103 & 91 & 128 \\
\hline
\end{tabular}


Table 2 Prevalence of Schistosoma mansoni categorized by age and sex

\begin{tabular}{|c|c|c|c|c|c|c|}
\hline Variable & & Number examined & Prevalence $(\%, n)$ & $95 \% \mathrm{Cl}$ & $x^{2}$ & $P$-value \\
\hline \multirow[t]{2}{*}{ Sex } & Female & 513 & $64.5 \%(331)$ & $60.3-68.6$ & 10.3523 & 0.001 \\
\hline & Male & 417 & $74.3 \%$ (310) & $69.9-78.3$ & & \\
\hline \multirow[t]{6}{*}{ Age (in years) } & $1-5$ & 71 & $54.9 \%(39)$ & $43.0-66.3$ & 105.61 & 0.001 \\
\hline & $6-15$ & 418 & $86.1 \%(360)$ & $82.5-89.1$ & & \\
\hline & $16-25$ & 119 & $57.1 \%(68)$ & $47.9-65.8$ & & \\
\hline & $26-35$ & 103 & $55.3 \%(57)$ & $45.4-64.7$ & & \\
\hline & $36-45$ & 91 & $51.7 \%(47)$ & $41.3-61.8$ & & \\
\hline & $\geq 46$ & 128 & $54.7 \%(170)$ & $45.8-63.2$ & & \\
\hline
\end{tabular}

d) belonging to the age group $36-45$ years $(\mathrm{aRR}=1.56$, 95\%CI: $1.31-1.84, P<0.001)$

e) and belonging to the age group $\geq 46$ years $(\mathrm{aRR}=$ 1.64, 95\%CI: $1.41-1.92, P<0.001)$

\section{Left liver lobe hepatomegaly, splenomegaly and their} associated factors or predictors

Data on left liver lobe measurements were available for 909 study participants. Overall, 51.9\% (472/909, 95\%CI: 48.7-55.2) of the study participants had enlarged and grossly enlarged left liver lobe. Of these, 79\% (373/472) and $21 \%(99 / 472)$ had enlarged and grossly enlarged left liver lobe respectively. In relation to sex, female study participants had higher prevalence of enlarged $(59.5 \%$ versus $40.5 \%)$ and grossly enlarged left liver lobe $(62.6 \%$ versus $\left.37.4 \%, X^{2}=10.0110, P<0.01\right)$. In the age groups, the age group 6-15 years recorded the highest prevalence of enlarged (49.6\%) and grossly enlarged (36.4\%) left liver lobe compared to other age groups $\left(x^{2}=\right.$ $29.1165, P<0.001)$. No statistical difference in prevalence of enlarged and grossly enlarged left liver lobe was observed in relation to $S$. mansoni infection status $(P=$ $0.82)$ and intensity of infection categories $(P=0.47)$.

Splenomegaly was detected in $31.2 \%(278 / 892$, $95 \%$ CI: 28.2-34.3) of the study participants whose data were available. Of these participants, 212/278 (76.3\%) and 66/ 278 (23.7\%) had moderately to marked enlarged splenomegaly. Male individuals recorded a higher prevalence of moderately (27.8\%) and markedly enlarged spleen $(9.5 \%)$ than the female individuals $\left(x^{2}=13.3959\right.$, $P<0.001)$. Similarly, there was a significance difference in prevalence of moderately and markedly enlarged spleen in relation to $S$. mansoni infection status. Individuals who had $S$. mansoni detectable eggs in their stool samples had a higher prevalence of moderately (26.1\%) and markedly enlarged spleen $(7.7 \%)\left(X^{2}=6.6121, P<\right.$ 0.037).

Hepatosplenomegaly (enlargement of both spleen and liver) was detected in $55.8 \%(155 / 895)$ of the study participants. Other morbidities detected were ascites $(0.2 \%)$, collateral veins (1.7\%) and gall bladder wall thickening (9.7\%).

On linear regression model using height adjusted ultrasound measurements of the left liver lobe as a continuous outcome variable for individuals who had detectable $S$. mansoni eggs in stool samples, the findings demonstrated that, the ultrasound adjusted left liver lobe measurements were significantly associated with being female $(P<0.03)$, being in the age groups $1-5$ years $(P<$ $0.013), 6-15$ years $(P<0.04)$ and $S$. mansoni intensity of infection $(P<0.034)$ (Table 7).

On bivariate analysis, factors associated with splenomegaly were mainly being male, belong to the age groups 16-25 years and 26-35 years and having

Table 3 Geometrical mean eggs per gram of faeces of S. mansoni stratified by age and sex

\begin{tabular}{|c|c|c|c|c|}
\hline Variables & & GMepg & $95 \% \mathrm{Cl}$ & T-test or F-test \\
\hline \multirow[t]{2}{*}{ Sex } & Female & 89.9 & $78.1-103.7$ & t-test $=-0.2887, P=0.77$ \\
\hline & Male & 81.4 & 70.4-94.0 & \\
\hline \multirow[t]{6}{*}{ Age groups (in years) } & $1-5$ & 91.9 & $58.8-143.5$ & F-test $=10.71, P<0.0001$ \\
\hline & $6-15$ & 108.6 & $95.2-124.0$ & \\
\hline & $16-25$ & 88.1 & $65.0-119.3$ & \\
\hline & $26-35$ & 61.7 & $43.2-88.1$ & \\
\hline & $36-45$ & 42.8 & $30.8-59.8$ & \\
\hline & $\geq 46$ & 49.3 & $37.3-65.0$ & \\
\hline
\end{tabular}


Table 4 Prevalence of Schistosoma mansoni based on point-of-care Circulating Cathodic Antigen (POC-CCA) test stratified by age and sex

\begin{tabular}{|c|c|c|c|c|c|c|}
\hline Variables & & No. examined & $\begin{array}{l}\text { Prevalence } \\
(\%, n)\end{array}$ & $95 \% \mathrm{Cl}$ & $x^{2}$ & $P$-value \\
\hline \multirow[t]{2}{*}{ Sex } & Female & 512 & $93.2 \%(477)$ & $90.6-95.1$ & 3.9839 & 0.046 \\
\hline & Male & 417 & $96.2 \%(401)$ & $93.8-97.6$ & & \\
\hline \multirow[t]{6}{*}{ Age (in years) } & $1-5$ & 71 & $95.8 \%(401)$ & 87.4-98.7 & 34.2723 & 0.001 \\
\hline & $6-15$ & 418 & $98.1 \%(410)$ & $96.2-99.0$ & & \\
\hline & $16-25$ & 119 & $91.6 \%(109)$ & $84.9-95.5$ & & \\
\hline & $26-35$ & 103 & $88.4 \%(91)$ & $80.4-93.3$ & & \\
\hline & $36-45$ & 91 & $97.8 \%(89)$ & $91.4-99.5$ & & \\
\hline & $\geq 46$ & 127 & $87.4 \%(111)$ & $80.3-92.2$ & & \\
\hline
\end{tabular}

detectable S. mansoni eggs in stool samples. Similarly, in the multivariable analysis, being male $(\mathrm{aRR}=1.15$, 95\%CI: $1.06-1.24, P<0.001)$, belong to the age group $16-25$ years $(\mathrm{aRR}=1.27,95 \% \mathrm{CI}: 1.05-1.54, P<0.02)$ and age group $26-35$ years $(\mathrm{aRR}=1.32,95 \% \mathrm{CI}: 1.08-1.61$, $P<0.01$ ) (Table 8 ).

\section{Comparison of the height adjusted mean deviations of organs in relation to infection with $S$. mansoni}

The mean deviations of ultrasound measurements of left liver lobe, spleen and portal vein were compared between individuals who were infected or uninfected with S. mansoni based on Kato Katz technique. Overall, the height-adjusted ultrasound measurements of the left liver lobe $(t=-0.7280, P=0.46)$ did not differ between the two groups. There was a significant difference in height-adjusted ultrasound measurements of the spleen $(\mathrm{t}=-2.0616, P<0.04)$ and portal vein $(\mathrm{t}=6.2970, P<$ 0.001 ) between individuals who were infected or not with S. mansoni. For the height-adjusted length of the spleen, individuals who were infected showed an enlargement of the spleen compared to uninfected individuals. For the portal vein, individuals who were infected had more dilated (increased diameter) portal vein diameter compared to uninfected individuals.

\section{Discussion}

The findings from this study indicated that Ijinga Island is highly endemic for $S$. mansoni infection. The prevalence of S. mansoni and its related intensity of infection is high in all age groups. Furthermore, our findings noted a difference in case detection of $S$. mansoni infection between Kato Katz (KK) technique and pointof-care Circulating Cathodic Antigen test. The POCCCA test detected more cases of the disease than KK technique. The findings further noted that S. mansoni related hepatosplenic morbidities are also common in all age groups and both sexes. Heavy intensity of $S$. mansoni infection was associated with hepatomegaly and splenomegaly. A number of demographic and

Table 5 Prevalence of periportal fibrosis in relation to demographic characteristics of the study participants, infection status and intensity of infection

\begin{tabular}{|c|c|c|c|c|c|c|}
\hline Variable & & No. examined & $\operatorname{PPF}(\%, n)$ & $95 \% \mathrm{Cl}$ & $x^{2}$ & $P$-value \\
\hline \multirow[t]{2}{*}{ Sex } & Female & 513 & $24.2 \%(124)$ & $20.7-28.1$ & 4.9559 & 0.026 \\
\hline & Male & 417 & $30.7 \%(128$ & $26.4-35.3$ & & \\
\hline \multirow[t]{6}{*}{ Age (in years) } & $1-5$ & 71 & $8.5 \%(6)$ & $3.7-17.8$ & 131.0316 & 0.001 \\
\hline & $6-15$ & 418 & $13.2 \%(55)$ & $10.2-16.8$ & & \\
\hline & $16-25$ & 119 & $31.1 \%(37)$ & $23.3-40.1$ & & \\
\hline & $26-35$ & 103 & $39.8 \%(41)$ & $30.6-49.6$ & & \\
\hline & $36-45$ & 91 & $47.3 \%(43)$ & $37.1-57.7$ & & \\
\hline & $\geq 46$ & 128 & $54.7 \%(70)$ & $45.8-63.2$ & & \\
\hline \multirow[t]{2}{*}{ S. mansoni infection status } & Infected & 641 & $24.3 \%(156)$ & $21.2-27.8$ & 7.9531 & 0.005 \\
\hline & Not infected & 289 & $33.2 \%(96)$ & $27.9-38.8$ & & \\
\hline \multirow[t]{3}{*}{ Intensity of infection } & $1-99$ & 354 & $25.9 \%(92)$ & $21.7-30.8$ & 1.7427 & 0.42 \\
\hline & $100-399$ & 204 & $21.1 \%(43)$ & $15.9-27.3$ & & \\
\hline & $\geq 400$ & 83 & $25.3 \%(21)$ & $16.9-35.9$ & & \\
\hline
\end{tabular}


Table 6 Risk factors associated with periportal fibrosis on ljinga Island, north-western Tanzania

\begin{tabular}{lllllll}
\hline Variable & RR & $95 \% \mathrm{Cl}$ & $P$-value & aRR & $95 \% \mathrm{Cl}$ & $P$-value \\
\hline Sex & & & & & & \\
$\begin{array}{l}\text { Female } \\
\text { Male }\end{array}$ & 1 & & & 1 & & \\
1.07 & $1.01-1.13$ & 0.03 & 1.08 & $1.02-1.16$ & 0.01
\end{tabular}

Age groups (in years)

\begin{tabular}{lllllll}
$1-5$ & 1 & & \multicolumn{1}{c}{1} & & \\
$6-15$ & 1.05 & $0.94-1.16$ & 0.38 & 1.06 & $0.92-1.21$ & 0.39 \\
$16-25$ & 1.25 & $1.11-1.42$ & 0.001 & 1.23 & $1.05-1.44$ & 0.01 \\
$26-35$ & 1.36 & $1.21-1.55$ & 0.001 & 1.42 & $1.21-1.67$ & 0.001 \\
$36-45$ & 1.47 & $1.29-1.68$ & 0.001 & 1.56 & $1.31-1.84$ & 0.001 \\
$\geq 46$ & 1.59 & $1.41-1.79$ & 0.001 & 1.64 & $1.41-1.92$ & 0.001
\end{tabular}

Infection status

Uninfected 1

Infected $0.91 \quad 0.86-0.97 \quad 0.005 \quad-\quad \ldots \quad-$

Intensity of infection (epg)

\begin{tabular}{lllllll}
$1-99$ & 1 & & \multicolumn{1}{c}{1} & & \\
$100-399$ & 0.95 & $0.88-1.03$ & 0.19 & 1.00 & $0.94-1.08$ & 0.92 \\
$\geq 400$ & 0.99 & $0.89-1.10$ & 0.89 & 1.12 & $1.01-1.23$ & 0.02 \\
\hline
\end{tabular}

epidemiological factors were noted to be partly contributing to the development $S$. mansoni related hepatosplenic morbidities in the study area.

Findings on the prevalence of $S$. mansoni infection on Ijinga Island corroborate the results of previous studies which demonstrated that communities along the southern shore line of Lake Victoria and its Islands carry the highest burden of the disease [12, 13, 18, 19, 23, 35]. Our findings noted a very high prevalence using both
Table 8 Factors associated with splenomegaly

\begin{tabular}{lllllll}
\hline Variables & RR & $95 \% \mathrm{Cl}$ & $P$-value & aRR & $95 \% \mathrm{Cl}$ & $P$-value \\
\hline Sex & & & & & & \\
Female & 1 & & & & & \\
Male & 1.12 & $1.05-1.88$ & 0.001 & 1.15 & $1.06-1.24$ & 0.001
\end{tabular}

Age groups (in years)

$\begin{array}{lllllll}1-5 & 1 & & & 1 & & \\ 6-15 & 1.11 & 0.97-1.25 & 0.10 & 1.07 & 0.95-1.21 & 0.28 \\ 16-25 & 1.19 & 1.03-1.37 & 0.02 & 1.18 & 1.03-1.36 & 0.02 \\ 26-35 & 1.16 & 1.01-1.35 & 0.04 & 1.16 & 1.01-1.34 & 0.04 \\ 36-45 & 1.09 & 0.93-1.26 & 0.27 & 1.10 & 0.95-1.28 & 0.19 \\ \geq 46 & 1.02 & 0.89-1.18 & 0.73 & 1.02 & 0.89-1.17 & 0.74\end{array}$

Infection status

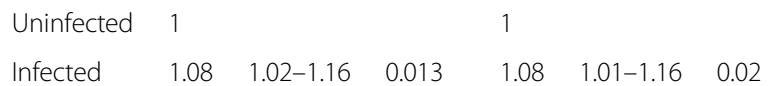

Intensity of infection (epg)

$\begin{array}{lllllll}1-99 & 1 & & & & \\ 100-399 & 1.00 & 0.92-1.09 & 0.98 & - & - & - \\ \geq 400 & 1.00 & 0.88-1.12 & 0.99 & - & - & -\end{array}$

KK technique and POC-CCA test. Based on KK technique, the prevalence of $S$. mansoni observed in this study was lower than the $78 \%$ [18] and $86.3 \%$ [13] recorded at the nearby Island of Ukerewe north-western Tanzania. Other previous studies in the same area have recorded lower prevalences $[19,23]$ than in the present study. A possible explanation could be the low rate of participation in previous MDA campaigns $(12.8 \%$ acc. to the questionnaire) of the Ijinga community. The age and sex differences in S. mansoni infection status recorded in

Table 7 Predictors of height adjusted left liver lobe size among residents of ljinga Island infected with S. mansoni

\begin{tabular}{|c|c|c|c|c|}
\hline Variables & $\beta$-coefficient & SE & $95 \% \mathrm{Cl}$ & $P$-value \\
\hline \multicolumn{5}{|l|}{ Sex } \\
\hline Female & 1 & & & \\
\hline Male & 0.24 & 0.1080857 & $0.03-0.45$ & 0.03 \\
\hline \multicolumn{5}{|l|}{ Age groups (in years) } \\
\hline $1-5$ & 0.8876 & 0.2503498 & $0.39-1.38$ & 0.001 \\
\hline $6-15$ & 0.3797 & 0.18022 & $0.03-0.733$ & 0.035 \\
\hline $16-25$ & -0.1455 & 0.2199547 & $-0.58-0.28$ & 0.51 \\
\hline $26-35$ & 1 & & & \\
\hline $36-45$ & 0.5855402 & 0.2361574 & $0.12-1.05$ & 0.013 \\
\hline$\geq 46$ & 0.305517 & 0.2153395 & $-0.12-0.72$ & 0.16 \\
\hline \multicolumn{5}{|c|}{ Schistosoma mansoni intensity (epg) } \\
\hline S.mansoni intensities & 0.004317 & 0.0002037 & $0.00003-0.008316$ & 0.034 \\
\hline
\end{tabular}

$\mathrm{R}^{2}=0.0313$ (adjusted $\mathrm{R}$-square), $\mathrm{F}=5.20, P<0.0001$

Linear regression model was constructed for height adjusted ultrasound measurements of the left liver lobe (organ size as continuous variable, i.e. deviations from mean) 
this present study were comparable to results of previous similar studies conducted in schistosomiasis endemic areas $[18,36,37]$. The variations in $S$. mansoni prevalence between age groups and sex is mainly explained by varying patterns of exposure to risk areas (water contacts) and development of acquired partial immunity $[13,36,38]$. The high prevalence of $>50 \%$ of $S$. mansoni infection in all the age groups based on KK technique and POC-CCA test indicate that Ijinga island is an old focus for $S$. mansoni transmission and for successful control of the disease and its related hepatosplenic morbidities, the entire community should receive at least two rounds of MDA per year according to WHO recommendation [39].

Of note to our findings is the high prevalence of $S$. mansoni infection in pre-school age children ( $\leq 6$ years) based on both KK technique and POC-CCA test. The age group had also high intensity of $S$. mansoni infection compared to the older age group. These findings indicate that, $S$. mansoni infections starts at an early age and with heavy infection intensities, the age group is likely to start developing hepatosplenic disease at early age. At the time these children reach maturity if left untreated, are likely to end up with severe hepatosplenic disease [40]. Similar studies in East Africa have recently reported a high prevalence and intensity of infection of $S$. mansoni in the same age group using both KK technique and POC-CCA test $[20,22]$. It is worthwhile noting that the use of KK technique alone in PSAC children results into underestimation of the prevalence of the infection. Therefore, it remains important to combine this technique with a more sensitive diagnostic technique such as POC-CCA test to increase the detection of true cases of the disease in this age group [20,41, 42]. In our present study, almost $41 \%$ of the pre-school children were missed by KK technique. Taking into account that the peadiatric formulation of praziquantel will be available in the near future [40,43], which will allow including of PSAC into the MDA program, it is important to include a more sensitive diagnostic test for case detection in this age group and for monitoring of drug efficacy [40].

The results of the present study show that ultrasound detectable hepatosplenic morbidities are common among our study population and almost exclusively are attributed to $S$. mansoni infection. Our results confirm the findings of the previous studies conducted in the same region which noted different patterns of organ morbidities in communities living along the shoreline of the lake and on islands $[12,13,18,21]$. The common hepatosplenic morbidities detected in the present study population were periportal fibrosis (PPF), hepatomegaly, splenomegaly, hepatosplenomegaly and gall bladder wall thickening. The prevalence of PPF observed in the present study was lower than the $41 \%$ [18] and 42\% [33] reported from Kome and Msozi village at Ukerewe district respectively. However, other similar studies in the same region have reported lower prevalences than what was recorded in this area [12, 21]. In the same region, hepatomegaly and splenomegaly related to $S$. mansoni infection is a common observation, for instance on Ukerewe Island, 35 and $80 \%$ of the studied population had left liver lobe hepatomegaly and splenomegaly [13]. In the nearby region of Mara, 28.5 and $29.6 \%$ of the study participants had splenomegaly and hepatomegaly [21] while on Kome Island, 68 and 55\% of the adult individuals were ultrasonographically detected to have hepatomegaly and splenomegaly [33]. Authors in previous similar studies have described a number of demographic and epidemiologic factors which partly could explain the variation in prevalence of ultrasound detectable $S$. mansoni related morbidities between communities living in different transmission settings [11, 12, 36, 37].

Interestingly, our findings noted a degree of hepatosplenic morbidities among children aged $<5$ years. The main hepatosplenic morbidities observed in the children was mainly left liver lobe enlargement, splenomegaly and only a small proportion of the children had signs of PPF. It should be noted that at such young age, is difficult to precisely classify the liver image pattern/ grades as detected by ultrasound [22]. An intensive inflammatory response due to the accumulation of $S$. mansoni eggs in the liver tissue, especially the left liver lobe could partly explain the liver size enlargement in this age group [7, 38]. Studies have reported an association between the enlargement of the left liver lobe and $S$. mansoni infection and its intensity [11, 36, 37]. The main concern here, is the development of $S$. mansoni related hepatosplenic morbidities at such young age and the fact that, to date this age group is not considered for MDA with praziquantel $[40,43,44]$. At the time when these children will be attending primary school, they are at risk of having already developed advanced stages of hepatosplenic disease. A recent study from Uganda, has pointed out the growing problem of hepatosplenic disease among pre-school aged children living in different transmission settings for S. mansoni infection [22]. In this study, PSAC were noticed to have already developed liver image grades suggestive of PPF (Symmer's pipe stems fibrosis) at six years of age [22]. This observation is commonly seen in adult population with long-standing infection with S. mansoni [18, 45]. Cumulatively, our observation and that of other authors in Uganda [22] on the development of hepatosplenic disease in this young age group suggest that the period from infection to the development of hepatosplenic morbidities is short compared to previous knowledge based on adult. It seems that $S$. mansoni hepatosplenic morbidities in the PSAC have been underestimated if not overlooked in many studies. It is 
high time now for this age group to be considered for appropriate treatment [44].

In the present study, the main risk factors associated with PPF were being a male, aged above 16 years and having heavy intensity of infection. The association of male sex with PPF mainly depicts the level of exposure and high infection intensity observed among male individuals in endemic areas [14, 18, 37]. The relationship between increased age and observation of chronic hepatosplenic morbidities in older ages mainly signifies that, $S$. mansoni is a chronic infection which requires time for the overt related morbidities such as PPF, hepatomegaly and splenomegaly to develop [11, 14, 37]. Risk factors such as village of residence, age, occupation (being fisherman), being male has been shown by previous studies to be associated with PPF in $S$. mansoni endemic areas $[11,36,37]$. Heavy intensity of $S$. mansoni infection was also noted in this present study to be associated with PPF. Similar studies have repeatedly described this observation in individuals with severe PPF grades [14, 37].

On the other hand, our findings noted a significant correlation between the heights adjusted length of the left liver lobe with $S$. mansoni intensity of infection, female individuals and age groups. Similarly, young age groups, having detectable S. mansoni eggs in stool sample and being male were associated with enlargement of the spleen. These explanatory factors have been previous described to interact in causing S. mansoni related hepatosplenic disease characterized by hepatomegaly, splenomegaly and hepatosplenomegaly in different settings [11, $36,37]$. In endemic areas, male individuals and children in young age groups are more exposed to water sources than other members of their communities and therefore accumulate heavy intensities of infection with increasing age. This partly explains the high prevalence of some of the hepatosplenic morbidities, for instance PPF and left liver lobe hepatomegaly among male individuals compared to females [14]. In the young age groups, left liver lobe hepatomegaly and splenomegaly have been noted to correlate with heavy intensities of $S$. mansoni infection $[22,36,37]$. It is worthwhile noting that, in schistosomiasis endemic areas, P. falciparum malaria is also a cause of hepatomegaly and splenomegaly and differential diagnosis especially in young age groups is a requirement [46].

The following limitation have to be discussed when interpretation of our results is done. The use of single stool sample for examination may have underestimated the intensity of $S$. mansoni infection taking into account the day to day variability of parasites eggs output. Also, we did not screen for malaria parasites especially in the young age groups, which could confound our findings on the enlargement of the left liver lobe and spleen.
The prevalence of Hepatitis B surface antigen positive chronic hepatitis $\mathrm{B}$ among the adult population of Tanzania is about 7\% [47]. It has to be assumed that coinfections with $S$. mansoni are common and chronic viral hepatitis contributes to the severity of liver disease. Nevertheless, the ultrasound patterns of liver cirrhosis or mixed pathology were not detected among our study participants. A screening for HBs-antigen would have required to take a blood sample what we considered to be an obstacle for a high rate of voluntary participation.

Our findings reflect the situation of schistosomiasis in many of the communities living on the islands of Lake Victoria, north-western Tanzania and we consider the conclusions drawn as transferable.

\section{Conclusion}

Our findings indicate that, S. mansoni infection and its related hepatosplenic morbidities are prevalent in the study area and the hepatosplenic morbidities show an association with with age and sex. The prevalence of S. mansoni infection was above $50 \%$ in all age groups and the youngest age groups had higher intensities of infection. Hepatosplenic morbidities were also detected among the children below $<5$ years of age. Based on the observed prevalence of the infection in all age groups, the entire community of Ijinga Island including pre-school children needs to be targeted for mass drug administration with praziquantel. We propose two to three rounds of MDA per year to reduce the prevalence and then revise to single annual dose for the entire community.

\section{Abbreviations}

KK: Kato Katz technique; MDA: Mass Drug Administration; POC-CCA: Point-ofcare Circulating Cathodic Antigen; PPF: Periportal fibrosis; PSCA: Pre-school aged children; SAC: School aged children; WHO: World Health Organization

\section{Acknowledgements}

We thank the study participants from the study village of ljinga Island for their participation in the study. We are grateful to the laboratory technicians' team from the National Institute for Medical Research and Catholic University of Health and Allied Sciences.

\section{Authors' contributions}

AM, AF and HDM designed the study, participated in data collection and analysis, and drafted the first version of the manuscript. UZ and GMK participated in the data collection. All authors read and approved the final manuscript, contributed to the critical review and made substantial contribution to it.

\section{Funding}

This work was supported by Rexroth Foundation, Germany and German Academic Exchange Service (Deutscher Akademischer Austauschdienst (DAAD), number 57141273 (https://www.daad.de/de/). The funding organizations did not have any influence on the study design, data collection, data analysis, interpretation of the results or writing of the manuscript. The contents of the manuscript are solely the responsibility of the authors and do not necessarily represent the official views of the supporting offices.

\section{Availability of data and materials}

The datasets collected and/or analyzed during the current study are available from the corresponding author upon reasonable request. However, we did 
not receive permission to share the raw data from the institution review boards.

\section{Ethics approval and consent to participate}

Ethical approval was obtained from the National Institutional Ethical Review Board (Cert. no.MR/53/100/434), Lake Zone Institutional Review Board unde the National Institute for Medical Research, Tanzania. Further ethical approval was thought from the Catholic University of Health and Allied Sciences, Ethical Review Committee (cert. no. CREC/156/2016). The study received government authorization from the regional and district administrative authorities of Mwanza region and Magu district. Swahili translated consent and assent (for children aged 8-16 years) were used to obtained participant's permission to participate in the study. Parental written informed consent was thought for all the children aged 3-16 years who participated in the study. For illiterate participants, a thumb print was used to sign the assent and consent form after they received a clear oral description of the study objectives and the treatment.

\section{Consent for publication}

Not applicable.

\section{Competing interests}

The authors declare that they have no competing interests.

\section{Author details}

'Department of Tropical Medicine, Klinikum Wuerzburg Mitte gGmbH, Medical Mission Hospital, Salvatorstrasse 7, 97074 Wuerzburg, Germany. ${ }^{2}$ Medical Mission Institute, Salvatorstrasse 7, 97074 Wuerzburg, Germany. ${ }^{3}$ National Institute for Medical Research, P.O. Box 1462, Mwanza, Tanzania ${ }^{4}$ School of Medicine, Department of Medical Parasitology, P.O. Box 1464, Mwanza, Tanzania.

Received: 24 May 2019 Accepted: 10 September 2019 Published online: 07 October 2019

\section{References}

1. Steinmann P, Keiser J, Bos R, Tanner M, Utzinger J. Schistosomiasis and water resources development: systematic review, meta-analysis, and estimates of people at risk. Lancet Infect Dis. 2006;6(7):411-25.

2. Global Burden of Disease Study 2013 Collaborators. Global, regional, and national incidence, prevalence, and years lived with disability for 301 acute and chronic diseases and injuries in 188 countries, 19902013: a systematic analysis for the global burden of disease study 2013. Lancet. 2015;386(9995):743-800.

3. van der Werf MJ, de Vlas SJ, Brooker S, Looman CW, Nagelkerke NJ, Habbema JDF, Engels D. Quantification of clinical morbidity associated with schistosome infection in sub-Saharan Africa. Acta Trop. 2003;86(2):125-39.

4. Hotez PJ, Kamath A. Neglected tropical diseases in sub-saharan Africa: review of their prevalence, distribution, and disease burden. PLoS Negl Trop Dis. 2009:3(8):e412

5. Mazigo HD, Nuwaha F, Kinung'hi SM, Morona D, Pinot de Moira A, Wilson S, Heukelbach J, Dunne DW. Epidemiology and control of human schistosomiasis in Tanzania. Parasit Vect. 2012;5:274.

6. King $\mathrm{CH}$, Dangerfield-Cha M. The unacknowledged impact of chronic schistosomiasis. Chronic Illiness. 2008;4(1):65-79.

7. Dunne DW, Pearce EJ. Immunology of hepatosplenic schistosomiasis mansoni: a human perspective. Micro Infect. 1999;1(7):553-60.

8. Richter J, Hatz C, Campagne G, Bergquist N, Jenkins JM. Ultrasound in schistosomiasis: a practical guide to the standard use of ultrasonography for assessment of schistosomiasis-related morbidity: second international workshop, October 22-26 1996, Niamey, Niger. Geneva: Tropical Diseases Research, World Health Organization; 2000.

9. Richter J, Poggensee G, Helling-Giese G, Kjetland E, Chitsulo L, Koumenda N, Gundersen SG, Krantz I, Feldmeier H. Transabdominal ultrasound for the diagnosis of Schistosoma haematobium infection of the upper female genital tract: a preliminary report. Trans R Soc Trop Med Hyg. 1995;89(5):500-1.

10. Warren KS. Hepatosplenic schistosomiasis mansoni: an immunologic disease. Bullettin of the New York Academy of Medicine. 1975;51(4):545-50.

11. Booth M, Vennervald BJ, Kabatereine NB, Kazibwe F, Ouma JH, Kariuki CH, Muchiri E, Kadzo H, Ireri E, Kimani G, et al. Hepatosplenic morbidity in two neighbouring communities in Uganda with high levels of Schistosoma mansoni infection but very different durations of residence. Trans $\mathrm{R}$ Soc Trop Med Hyg. 2004;98(2):125-36.

12. Mazigo HD, Dunne DW, Morona D, Lutufyo TE, Kinung'hi SM, Kaatano G, Nuwaha F. Periportal fibrosis, liver and spleen sizes among S. mansoni mono or co-infected individuals with human immunodeficiency virus-1 in fishing villages along Lake Victoria shores, North-Western, Tanzania. Parasit Vect. 2015;8:260

13. Kardorff R, Gabone RM, Mugashe C, Obiga D, Ramarokoto CE, Mahlert C, Spannbrucker N, Lang A, Gunzler V, Gryseels B, et al. Schistosoma mansonirelated morbidity on Ukerewe Island, Tanzania: clinical, ultrasonographical and biochemical parameters. Trop Med Int Health. 1997;2(3):230-9.

14. Mohamed-Ali Q, Elwali NE, Abdelhameed AA, Mergani A, Rahoud S, Elagib KE, Saeed OK, Abel L, Magzoub MM, Dessein AJ. Susceptibility to periportal (Symmers) fibrosis in human Schistosoma mansoni infections: evidence that intensity and duration of infection, gender, and inherited factors are critical in disease progression. J Infect Dis. 1999;180(4):1298-306.

15. Rollinson D, Knopp S, Levitz S, Stothard JR, Tchuem Tchuente LA, Garba A, Mohammed KA, Schur N, Person B, Colley DG, et al. Time to set the agenda for schistosomiasis elimination. Acta Trop. 2013;128(2):423-40.

16. Olsen A, Kinung'hi S, Magnussen P. Schistosoma mansoni infection along the coast of Lake Victoria in Mwanza region, Tanzania. Am J Trop Med Hyg. 2015;92(6):1240-4. https://doi.org/10.4269/ajtmh.14-0676.

17. Tanzania National Bureau of Statistics: Tanzania Populations census 2012Tanzania Government 2012.

18. Malenganisho WL, Magnussen P, Friis H, Siza J, Kaatano G, Temu M, Vennervald BJ. Schistosoma mansoni morbidity among adults in two villages along Lake Victoria shores in Mwanza District, Tanzania. Trans R Soc Trop Med Hyg. 2008;102(6):532-41.

19. Mugono M, Konje E, Kuhn S, Mpogoro FJ, Morona D, Mazigo HD. Intestinal schistosomiasis and geohelminths of Ukara Island, North-Western Tanzania: prevalence, intensity of infection and associated risk factors among school children. Parasit Vectors. 2014;7:612

20. Ruganuza DM, Mazigo HD, Waihenya R, Morona D, Mkoji GM. Schistosoma mansoni among pre-school children in Musozi village, Ukerewe Island, North-Western-Tanzania: prevalence and associated risk factors. Parasit Vectors. 2015;8:377.

21. Mazigo HD, Nuwaha F, Dunne DW, Kaatano GM, Angelo T, Kepha S, Kinung'hi SM. Schistosoma mansoni infection and its related morbidity among adults living in selected villages of Mara region, North-Western Tanzania: a cross-sectional exploratory study. Korean J Parasitol. 2017; 55(5):533-40.

22. Nalugwa A, Nuwaha F, Tukahebwa EM, Olsen A. Schistosoma mansoniassociated morbidity among preschool-aged children along the shores of Lake Victoria in Uganda. Trop Med Infect Dis. 2017;2(4).

23. Mazigo HD, Dunne DW, Wilson S, Kinung Hi SM, de Moira A, Jones FM, Morona D, Nuwaha F. Co-infection with Schistosoma mansoni and human immunodeficiency Virus-1 (HIV-1) among residents of fishing villages of North-Western Tanzania. Parasit Vect. 2014;7(1):587.

24. Mazigo HD, Waihenya R, Lwambo NJ, Mnyone LL, Mahande AM, Seni J, Zinga M, Kapesa A, Kweka EJ, Mshana SE, et al. Co-infections with Plasmodium falciparum, Schistosoma mansoni and intestinal helminths among schoolchildren in endemic areas of northwestern Tanzania. Parasit Vectors. 2010;3:44.

25. World Health Organization: WHO: Soil-transmitted helminthiases. Eliminating soil-transmitted helminthiases as a public health problem in children: progress report 2001-2010 and strategic plan 2011-2020 2012, World Health Organisation; 2012, Geneva 2010.

26. World Health Organization: Report of an informal consultation os schistosomiasis control. 2011 p. 1-67. . 2011.

27. Zou L, Ruan S. Schistosomiasis transmission and control in China. Acta Trop. 2015;143:51-7.

28. Mwanza region socio-economic profile. The United Republic of Tanzania, 1997. www.tzonline.org/pdf/Mwanza.pdf.

29. Naing $L$, Winn T, Rusli BN. Practical issues in calculating the sample size for prevalence studies. Arch Orofac Sci. 2006;2006

30. Katz N, Chaves A, Pellegrino J. A simple device for quantitative stool thicksmear technique in schistosomiasis mansoni. Rev Inst Med Trop Sao Paulo. 1972;14(6):397-400.

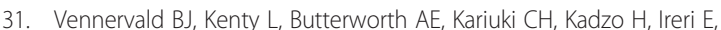
Amaganga C, Kimani G, Mwatha J, Otedo A, et al. Detailed clinical and 
ultrasound examination of children and adolescents in a Schistosoma mansoni endemic area in Kenya: hepatosplenic disease in the absence of portal fibrosis. Trop Med Int Health. 2004;9(4):461-70.

32. World Health Organization.: Basic laboratory methods in medical parasitology. 1991.

33. Kaatano GM, Min DY, Siza JE, Yong TS, Chai JY, Ko Y, Chang SY, Changalucha JM, Eom KS, Rim HJ. Schistosoma mansoni-related Hepatosplenic morbidity in adult population on Kome Island, Sengerema District, Tanzania. Korean J Parasitol. 2015;53(5):545-51.

34. King $\mathrm{CH}$, Magak P, Salam EA, Ouma JH, Kariuki HC, Blanton RE. Measuring morbidity in schistosomiasis mansoni: relationship between image pattern, portal vein diameter and portal branch thickness in large-scale surveys using new WHO coding guidelines for ultrasound in schistosomiasis. Trop Med Int Health. 2003;8(2):109-17.

35. Mazigo H, Dunne DW, Kinung'hi SM, Nuwaha F. Praziquantel efficacy against Schistosoma mansoni among HIV-1 infected and uninfected adults living in fishing villages along Lake Victoria, Northwest Tanzania. Infect Dis Poverty. 2014;3:47.

36. Boisier P, Ramarokoto CE, Ravoniarimbinina P, Rabarijaona L, Ravaoalimalala V. Geographic differences in hepatosplenic complications of schistosomiasis mansoni and explanatory factors of morbidity. Tropical Med Int Health. 2001:6(9):699-706.

37. Berhe N, Myrvang B, Gundersen SG. Intensity of Schistosoma mansoni, hepatitis B, age, and sex predict levels of hepatic periportal thickening/ fibrosis (PPT/F): a large-scale community-based study in Ethiopia. Am J Trop Med Hyg. 2007;77(6):1079-86.

38. Gryseels B. Morbidity due to infection with Schistosoma mansoni: an update. Trop Geogr Med. 1992;44(3):189-200.

39. World Health Organization. Prevention and control of schistosomiasis and soil-transmitted helminthiasis. World Health Organ Tech Rep Ser. 2002:912:i.

40. Osakunor DNM, Woolhouse MEJ, Mutapi F. Paediatric schistosomiasis: what we know and what we need to know. PLoS Negl Trop Dis. 2018; 12(2):e0006144

41. Fuss A, Mazigo HD, Tappe D, Kasang C, Mueller A. Comparison of sensitivity and specificity of three diagnostic tests to detect Schistosome mansoni infections in school children in Mwanza region, Tanzania. PLoS One. 2018:13(8):e0202499.

42. Mazigo HD, Kepha S, Kinung'hi SM. Sensitivity and specificity of point-ofcare circulating cathodic antigen test before and after praziquantel treatment in diagnosing Schistosoma mansoni infection in adult population co-infected with human immunodeficiency virus-1, North-Western Tanzania. Arch Public Health. 2018;76:29.

43. Mduluza T, Mutapi F. Putting the treatment of paediatric schistosomiasis into context. Infect Dis Poverty. 2017:6(1):85.

44. Stothard JR, Sousa-Figueiredo JC, Betson M, Bustinduy A, R-R J. Schistosomiasis in African infants and preschool children:let them now be treated. Trends Parasitol. 2013;29(4):197-205

45. Kabatereine N, Kemijumbi J, Ouma J, Kariuki H, Richter J, Kadzo H, Madsen H, Butterworth A, Ørnbjerg N, Vennervald B. Epidemiology and morbidity of Schistosoma mansoni infection in a fishing community along Lake Albert in Uganda. Trans R Soc Trop Med Hyg. 2004;98(12):711-8.

46. Wilson S, Jones FM, Mwatha JK, Kimani G, Booth M, Kariuki HC, Vennervald BJ, Ouma JH, Muchiri E, Dunne DW. Hepatosplenomegaly associated with chronic malaria exposure: evidence for a pro-inflammatory mechanism exacerbated by schistosomiasis. Parasite Immunol. 2009;31(2):64-71.

47. Mueller $A$, et al. Prevalence of hepatitis B virus infection among health care workers in a tertiary hospital in Tanzania. BMC Infect Dis. 2015;15:386. https://doi.org/10.1186/s12879-015-1129-z.

\section{Publisher's Note}

Springer Nature remains neutral with regard to jurisdictional claims in published maps and institutional affiliations.

Ready to submit your research? Choose BMC and benefit from:

- fast, convenient online submission

- thorough peer review by experienced researchers in your field

- rapid publication on acceptance

- support for research data, including large and complex data types

- gold Open Access which fosters wider collaboration and increased citations

- maximum visibility for your research: over $100 \mathrm{M}$ website views per year

At $\mathrm{BMC}$, research is always in progress.

Learn more biomedcentral.com/submissions 